\title{
Bayesian Tracking with Auxiliary Discrete Processes. Application to Detection and Tracking of Objects with Occlusions
}

\author{
Patrick Pérez ${ }^{1}$ and Jaco Vermaak ${ }^{2}$ \\ ${ }^{1}$ IRISA/INRIA, Rennes, France \\ perez@irisa.fr \\ ${ }^{2}$ Cambridge Univ. Eng. Dpt, Cambridge, U.K. \\ jv211@eng.cam.ac.uk
}

\begin{abstract}
A number of Bayesian tracking models involve auxiliary discrete variables beside the main hidden state of interest. These discrete variables usually follow a Markovian process and interact with the hidden state either via its evolution model or via the observation process, or both. We consider here a general model that encompasses all these situations, and show how Bayesian filtering can be rigorously conducted with it. The resulting approach facilitates easy re-use of existing tracking algorithms designed in the absence of the auxiliary process. In particular we show how particle filters can be obtained based on sampling only in the original state space instead of sampling in the augmented space, as it is usually done. We finally demonstrate how this framework facilitates solutions to the critical problem of appearance and disappearance of targets, either upon scene entering and exiting, or due to temporary occlusions. This is illustrated in the context of color-based tracking with particle filters.
\end{abstract}

\section{Introduction and Motivation}

Visual tracking involves the detection and recursive localization of objects within video frames. Often, the state of interest, e.g., size and location of the object, is associated with auxiliary discrete variables. Such variables show up for instance within the state evolution model, e.g., when different types of dynamics can occur [3]. More often, such auxiliary variables are introduced in the observation model. It is the case for appearance models based on a set of key views [810] or silhouettes 81. Auxiliary variables are also used to handle partial or total occlusions [6] or mutual occlusions when jointly tracking multiple objects [510]. Finally, auxiliary variables can be used to assess the presence of tracked objects in the scene 94 . When a Bayesian tracking approach is used with such augmented models, either specific filters are derived based on the detailed form of the model at hand or the optimal filter of the joint model is simply used. In the latter case, a practical implementation might be unnecessarily costly due to the increased dimension of the joint space. Sequential Monte Carlo approximations (SMC) in the joint space are for instance used in 3448910 . 
The first contribution of this paper is to propose a general and unified framework to easily derive the optimal Bayesian filter for the augmented model based on the one for a model with no (or frozen) auxiliary variables. In practice, this allows the re-use of existing tracking architectures, with a reasonable computational overhead in case the discrete auxiliary variable only takes a small number of values. This approach allows us in particular to introduce a generic SMC architecture that relies on sampling in the main state space only. This is exposed in Section 2.

The problem of appearing and disappearing objects, whether it is upon entering and exiting the scene, or upon getting occluded by another object, is critical in visual tracking. As we mentioned above, the different forms of this problem have already been addressed in the past based on auxiliary hidden processes. The second contribution of this paper is to re-visit these problems using our generic framework. The resulting filters are implemented using the our generic SMC architecture. To handle occlusions, we introduce in Section 3 a binary visibility process that intervenes in the observation model. In this case, our generic approach allows us to derive a two-fold mixture filter that deal with temporary occlusions. In a similar fashion, we address the problem of "birth" and "death" of objects, which is crucial for multiple-object tracking, by introducing a binary existence process. This process impacts both the state evolution and the data model. The application of our approach leads in this case to a simple filter whose SMC approximation does not need to draw samples for the existence variable.

\section{Tracking with an Auxiliary Process}

\subsection{Modeling Assumptions}

For visual tracking, we are interested in recursively estimating the object state $\mathbf{x}_{t} \in \mathbb{R}^{n_{x}}$, which specifies the position of the object in the image plane and, possibly, other parameters such as its size and orientation, based on a sequence of observations $\mathbf{y}^{t} \doteq\left(\mathbf{y}_{1} \cdots \mathbf{y}_{t}\right)$. We assume in addition that a discrete auxiliary variable $a_{t}$ also has to be recursively inferred. This variable takes its values in a set of cardinality $M$ that we will denote by $\{0 \cdots M-1\}$ for convenience.

The complete set of unknowns at time $t$ is thus $\left\{\mathbf{x}_{t}, a_{t}\right\}$, for which we assume the following Markovian prior

$$
p\left(\mathbf{x}_{t}, a_{t} \mid \mathbf{x}_{t-1}, a_{t-1}\right)=p\left(\mathbf{x}_{t} \mid \mathbf{x}_{t-1}, a_{t}, a_{t-1}\right) p\left(a_{t} \mid a_{t-1}\right) .
$$

In other words, the state follows a Markov chain with its kernel parameterized by the current and previous values of the auxiliary variable, and the auxiliary process is a discrete Markov chain. Let $A=\left(\alpha_{j i}\right)$ be its $M \times M$ transition matrix, with $\alpha_{j i} \doteq p\left(a_{t}=i \mid a_{t-1}=j\right)$. For brevity, we will also use the notation

$$
p_{j i}\left(\mathbf{x}_{t} \mid \mathbf{x}_{t-1}\right) \doteq p\left(\mathbf{x}_{t} \mid \mathbf{x}_{t-1}, a_{t}=i, a_{t-1}=j\right) .
$$

As for the observation model, we assume in the normal way that the image data at successive instances are independent conditional on the hidden variables, i.e., $p\left(\mathbf{y}_{t} \mid \mathbf{x}_{t}, a_{t}, \mathbf{y}^{t-1}\right)=p\left(\mathbf{y}_{t} \mid \mathbf{x}_{t}, a_{t}\right)$. For notational convenience we will denote 


$$
p_{i}\left(\mathbf{y}_{t} \mid \mathbf{x}_{t}\right) \doteq p\left(\mathbf{y}_{t} \mid \mathbf{x}_{t}, a_{t}=i\right) .
$$

The graphical model of the resulting joint distribution $p\left(\mathbf{x}_{0: t}, a_{0: t}, \mathbf{y}_{1: t}\right)$ is given in Fig. 1,

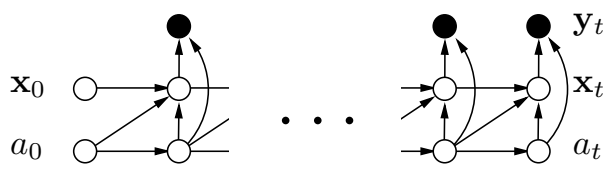

Fig. 1. Graphical model of the joint distribution $p\left(\mathbf{x}_{0: t}, a_{0: t}, \mathbf{y}_{1: t}\right)$ over the state, auxiliary and measurement processes

\subsection{Bayesian Filter}

For tracking, we are interested in recursively estimating the joint filtering distribution

$$
p\left(\mathbf{x}_{t}, a_{t} \mid \mathbf{y}^{t}\right)=p\left(\mathbf{x}_{t} \mid a_{t}, \mathbf{y}^{t}\right) p\left(a_{t} \mid \mathbf{y}^{t}\right),
$$

from which the marginal filtering distribution can be deduced as

$$
p\left(\mathbf{x}_{t} \mid \mathbf{y}^{t}\right)=\sum_{i} p\left(\mathbf{x}_{t}, a_{t}=i \mid \mathbf{y}^{t}\right)=\sum_{i} p_{i}\left(\mathbf{x}_{t} \mid \mathbf{y}^{t}\right) \xi_{i, t},
$$

where we used the notation

$$
\begin{aligned}
p_{i}\left(\mathbf{x}_{t} \mid \mathbf{y}^{t}\right) & \doteq p\left(\mathbf{x}_{t} \mid a_{t}=i, \mathbf{y}^{t}\right) \\
\xi_{i, t} & \doteq p\left(a_{t}=i \mid \mathbf{y}^{t}\right) .
\end{aligned}
$$

Similar to our previous notation, we will now use the distribution subscript $i$ to indicate conditioning with respect to the current auxiliary variable set to $i$, and the distribution subscript $j i$ for conditioning on $i$ and $j$ being the current and previous values of the auxiliary variable.

We will first show how to compute the $M$ conditional state posteriors $p_{i}\left(\mathbf{x}_{t} \mid \mathbf{y}^{t}\right)$. First note that

$$
p_{i}\left(\mathbf{x}_{t} \mid \mathbf{y}^{t}\right)=\frac{p_{i}\left(\mathbf{x}_{t}, \mathbf{y}_{t} \mid \mathbf{y}^{t-1}\right)}{p_{i}\left(\mathbf{y}_{t} \mid \mathbf{y}^{t-1}\right)} .
$$

The numerator can be expressed as

$$
p_{i}\left(\mathbf{x}_{t}, \mathbf{y}_{t} \mid \mathbf{y}^{t-1}\right)=\sum_{j} p_{j i}\left(\mathbf{x}_{t}, \mathbf{y}_{t} \mid \mathbf{y}^{t-1}\right) p\left(a_{t-1}=j \mid a_{t}=i, \mathbf{y}^{t-1}\right),
$$

with 


$$
\begin{aligned}
p_{j i}\left(\mathbf{x}_{t}, \mathbf{y}_{t} \mid \mathbf{y}^{t-1}\right)=p_{i}\left(\mathbf{y}_{t} \mid \mathbf{x}_{t}\right) p_{j i}\left(\mathbf{x}_{t} \mid \mathbf{y}^{t-1}\right) & \\
= & p_{i}\left(\mathbf{y}_{t} \mid \mathbf{x}_{t}\right) \int p_{j i}\left(\mathbf{x}_{t} \mid \mathbf{x}_{t-1}\right) p_{j}\left(\mathbf{x}_{t-1} \mid \mathbf{y}^{t-1}\right) d \mathbf{x}_{t-1},
\end{aligned}
$$

and

$$
\begin{aligned}
p\left(a_{t-1}=j \mid a_{t}=i, \mathbf{y}^{t-1}\right) \doteq & \tilde{\alpha}_{j i, t} \\
& \propto p\left(a_{t}=i \mid a_{t-1}=j, \mathbf{y}^{t-1}\right) p\left(a_{t-1}=j \mid \mathbf{y}^{t-1}\right) .
\end{aligned}
$$

Based on the conditional independence structure of the model, one can show that the first term on the right hand side is independent of $\mathbf{y}^{t-1}$. We thus obtain, after normalization,

$$
\tilde{\alpha}_{j i, t}=\frac{\alpha_{j i} \xi_{j, t-1}}{\sum_{k} \alpha_{k i} \xi_{k, t-1}}
$$

The predictive likelihood in the denominator of (8) is

$$
p_{i}\left(\mathbf{y}_{t} \mid \mathbf{y}^{t-1}\right)=\sum_{j} \tilde{\alpha}_{j i, t} \int p_{j i}\left(\mathbf{x}_{t}, \mathbf{y}_{t} \mid \mathbf{y}^{t-1}\right) d \mathbf{x}_{t} .
$$

The filtering distribution in (5) is then a mixture of the $M$ conditional filtering distributions, i.e.,

$$
p_{i}\left(\mathbf{x}_{t} \mid \mathbf{y}^{t}\right)=\frac{\sum_{j} \tilde{\alpha}_{j i, t} p_{j i}\left(\mathbf{x}_{t}, \mathbf{y}_{t} \mid \mathbf{y}^{t-1}\right)}{p_{i}\left(\mathbf{y}_{t} \mid \mathbf{y}^{t-1}\right)},
$$

each of which is obtained by combining $M$ optimal Bayesian filters to compute (10) and (13).

We still need the marginal posterior of the auxiliary variable, $p\left(a_{t} \mid \mathbf{y}^{t}\right)$, to compute the weights $\xi_{i, t}$ in the mixture of (5). We have

$$
\xi_{i, t} \propto p_{i}\left(\mathbf{y}_{t} \mid \mathbf{y}^{t-1}\right) \sum_{j} p\left(a_{t}=i \mid a_{t-1}=j, \mathbf{y}^{t-1}\right) \xi_{j, t-1} .
$$

Since the first factor in the sum is independent of $\mathbf{y}^{t-1}$, we finally obtain, after normalization

$$
\xi_{i, t}=\frac{p_{i}\left(\mathbf{y}_{t} \mid \mathbf{y}^{t-1}\right) \sum_{j} \alpha_{j i} \xi_{j, t-1}}{\sum_{k} p_{k}\left(\mathbf{y}_{t} \mid \mathbf{y}^{t-1}\right) \sum_{j} \alpha_{j k} \xi_{j, t-1}} .
$$

We present below an algorithmic summary of the operations at time $t$ of the generic algorithm.

- Input: $p_{i}\left(\mathbf{x}_{t-1} \mid \mathbf{y}^{t-1}\right)$ and $\left(\xi_{i, t-1}\right)$ for $i=0 \cdots M-1$.

1. Compute $\tilde{\alpha}_{j i, t}$ as in (12), for $i=0 \cdots M-1$.

2. Compute the $M^{2}$ distributions $p_{j i}\left(\mathbf{x}_{t}, \mathbf{y}_{t} \mid \mathbf{y}^{t-1}\right)$ as in (10), for $i, j=0 \cdots M-$ 1.

3. Compute the $M$ measurement prediction distributions $p_{i}\left(\mathbf{y}_{t} \mid \mathbf{y}^{t-1}\right)$ as in (13), for $i=0 \cdots M-1$. 
4. Compute the $M$ updated filtering distributions $p_{i}\left(\mathbf{x}_{t} \mid \mathbf{y}^{t}\right)=$ as in (14), for $i=0 \cdots M-1$.

5. Compute the marginal posterior probability vector $\left(\xi_{i, t}\right)_{i=0 \cdots M-1}$ of the auxiliary variable as in (16).

- Output: distributions $p_{i}\left(\mathbf{x}_{t} \mid \mathbf{y}^{t}\right)$ and weights $\xi_{i, t}$.

At each time step, $M^{2}$ "elementary" filtering operations are required (step 2 ), one per possible occurrence of the pairing $\left(a_{t}, a_{t-1}\right)$. In practice, not all $M^{2}$ values may be admissible, in which case the number of elementary filtering operations at each time step is reduced accordingly. As we will see, specificities of the model under consideration might also permit further computational savings.

The framework above is entirely general, both in terms of model ingredients (evolution and observation processes) and in terms of implementation. Regarding the latter, all existing techniques, whether exact or approximate, can be accommodated. If, for example, the filtering distributions $p_{i}\left(\mathbf{x}_{t} \mid \mathbf{y}^{t}\right)$ are to be represented by Gaussian mixtures, the mixtures components can be obtained by the Kalman filter for linear Gaussian models, and by the extended or unscented Kalman filters for non-linear and/or non-Gaussian models. For models of the latter kind it may sometimes be beneficial to adopt a particle representation, and use sequential importance sampling techniques to update the filtering distribution. This is especially true for the highly non-linear and multi-modal models used in visual tracking, hence the success of SMC techniques in the computer vision community. It is this type of implementation that we now consider.

\subsection{SMC Implementation}

For a general SMC implementation, we will consider proposal distributions of the form $q_{j i}\left(\mathbf{x}_{t} \mid \mathbf{x}_{t-1}, \mathbf{y}_{t}\right) \doteq q\left(\mathbf{x}_{t} \mid \mathbf{x}_{t-1}, a_{t}=i, a_{t-1}=j, \mathbf{y}_{t}\right)$. Based on these proposals, different SMC architectures can be designed to approximate the generic algorithm of the previous section. We propose here an architecture that is based on systematic resampling. Assuming that each conditional posterior distribution $p_{i}\left(\mathbf{x}_{t-1} \mid \mathbf{y}^{t-1}\right)$ at time $t-1$ is approximated by a set $\left(\mathbf{s}_{i, t-1}^{(n)}\right)_{n=1 \cdots N}$ of $N$ equally weighted particles, we simply replace steps 2,3 and 4 in the algorithm of Section 2.2 by:

2. For $j=0 \cdots M-1$, for $i=0 \cdots M-1$

2a. Sample $N$ particles $\tilde{\mathbf{s}}_{j i, t}^{(n)} \sim q_{j i}\left(\mathbf{x}_{t} \mid \mathbf{s}_{j, t-1}^{(n)}, \mathbf{y}_{t}\right)$.

2b. Compute the normalized predictive weights

$$
\pi_{j i, t}^{(n)} \propto \frac{p_{j i}\left(\widetilde{\mathbf{s}}_{j i, t}^{(n)} \mid \mathbf{s}_{j, t-1}^{(n)}\right)}{q_{j i}\left(\tilde{\mathbf{s}}_{j i, t}^{(n)} \mid \mathbf{s}_{j, t-1}^{(n)}\right), \mathbf{y}_{t}} \text { with } \sum_{n} \pi_{j i, t}^{(n)}=1 .
$$

3. Approximate the $M$ predictive data likelihoods by

$$
p_{i}\left(\mathbf{y}_{t} \mid \mathbf{y}^{t-1}\right) \approx \sum_{j} \sum_{n} w_{j i, t}^{(n)},
$$


where, for $i, j=0 \cdots M-1$,

$$
w_{j i, t}^{(n)} \doteq \tilde{\alpha}_{j i, t} p_{i}\left(\mathbf{y}_{t} \mid \tilde{\mathbf{s}}_{j i, t}^{(n)}\right) \pi_{j i, t}^{(n)} .
$$

4. For $i=0 \cdots M-1$, draw $N$ particles $\mathbf{s}_{i, t}^{(n)}$ with replacement from the weighted set $\left(\tilde{\mathbf{s}}_{j i, t}^{(n)}, p_{i}\left(\mathbf{y}_{t} \mid \mathbf{y}^{t-1}\right)^{-1} w_{j i, t}^{(n)}\right)_{j, n}$ of $M \times N$ particles.

Steps 1 and 5 remain unchanged.

At each instant $t$, posterior expectations can be approximated using the final particle sets:

$$
\begin{aligned}
\mathbb{E}\left[\mathbf{x}_{t} \mid a_{t}=i, \mathbf{y}^{t}\right] & \approx \hat{\mathbf{x}}_{i, t} \doteq \frac{1}{N} \sum_{n} \mathbf{s}_{i, t}^{(n)} \\
\mathbb{E}\left[\mathbf{x}_{t} \mid \mathbf{y}^{t}\right] & \approx \hat{\mathbf{x}}_{t} \doteq \sum_{i} \xi_{i, t} \hat{\mathbf{x}}_{i, t} .
\end{aligned}
$$

If the proposal distribution does not depend on $a_{t}=i$, then step 2a can be performed $M$ times instead of $M^{2}$ times, providing particles sets $\left(\tilde{\mathbf{s}}_{j, t}^{(n)}\right)_{n}$ to be used in place of $\left(\tilde{\mathbf{s}}_{j i, t}^{(n)}\right)_{n}$ in the remainder of the algorithm.

Note that, contrary to standard SMC handling of models with auxiliary variables (e.g., 348910]), the proposed implementation restricts sampling to the original state space of interest, thus avoiding the sampling of the auxiliary variable.

\section{Appearance and Disappearance}

Most tracking algorithms assume he number of objects of interest to be constant in the sequence. However, in most cases objects of interest enter and exit the scene at arbitrary times. In addition, they can also disappear temporarily behind other occluding objects. In the latter case of occlusion, tracking should be continued blindly in the hope of locking back onto the objects when they re-appear. An object entering or exiting the scene should in contrast result in initiating or terminating tracking, respectively. In any case, these appearance and disappearance events, whether they are temporary or definitive, are themselves uncertain events. The associated concepts of "existence" and "visibility" should thus be treated jointly with the other unknowns within a probabilistic framework that can account for all the expected ambiguities. Exploiting the generic approach presented in the previous section, we propose to achieve this using two auxiliary binary processes. Although these two processes can be used jointly, we introduce them separately for the sake of clarity.

\subsection{Visibility Process}

Explicit introduction of an occlusion process within the Bayesian tracking framework was proposed in [5] and [10]. Both works, however, rely on specific modeling 

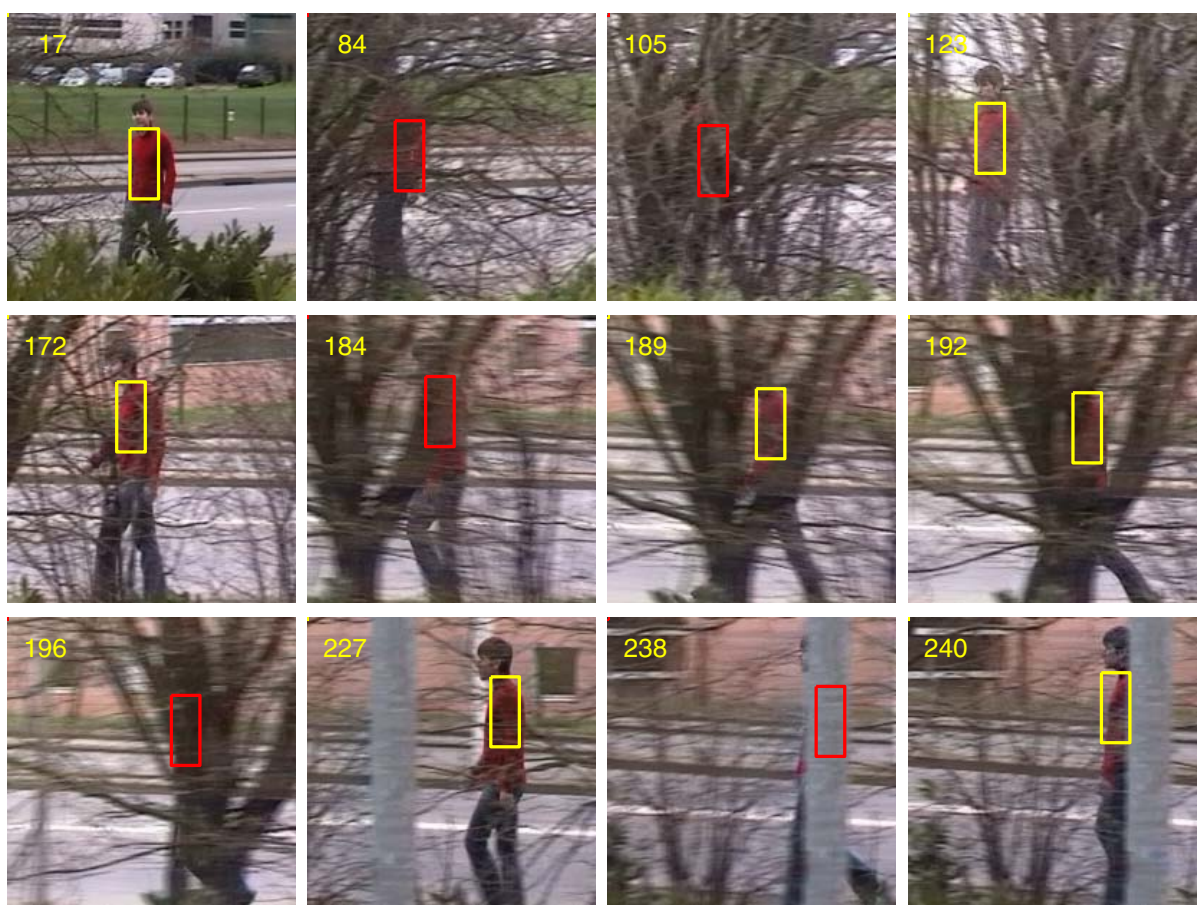

Fig. 2. Tracking under severe occlusions. The color-based tracker is initialized on the top of the walking person. The succession of occlusions caused by foreground trees is successfully handled thanks to the explicit modeling of visibility changes. The box corresponds to the MC approximation of the posterior state expectation. Its color is changed from yellow to red when the posterior visibility probability drops below 0.5 .

assumption (contour-based tracking in the former, luminance exemplars in the latter), and specific implementations (particle filter with partitioned importance sampling in the former vanilla bootstrap particle filter in the latter). In contrast, our approach relies on generic modeling assumptions and is independent of a specific implementation strategy, so that existing tracking architectures can be re-used. The occlusion modeling we propose can thus be used in conjunction with any Bayesian visual tracking technique, based for instance on the Kalman filter or one of its variants. In addition, using it within the SMC architecture of Section 2.3 allows restriction of the sampling to the object state space only.

Considering here only the case of complete occlusion, we introduce a binary visibility variable $v_{t}$ that indicates whether the object is visible $\left(v_{t}=1\right)$ or not $\left(v_{t}=0\right)$ in the image at time $t$. The Markov chain prior on this binary variable is completely defined by the occlusion and desocclusion probabilities, $\alpha_{10}$ and $\alpha_{01}$. The state evolution model is independent of the visibility variable, i.e.,

$$
p_{j i}\left(\mathbf{x}_{t} \mid \mathbf{x}_{t-1}\right)=p\left(\mathbf{x}_{t} \mid \mathbf{x}_{t-1}\right)
$$




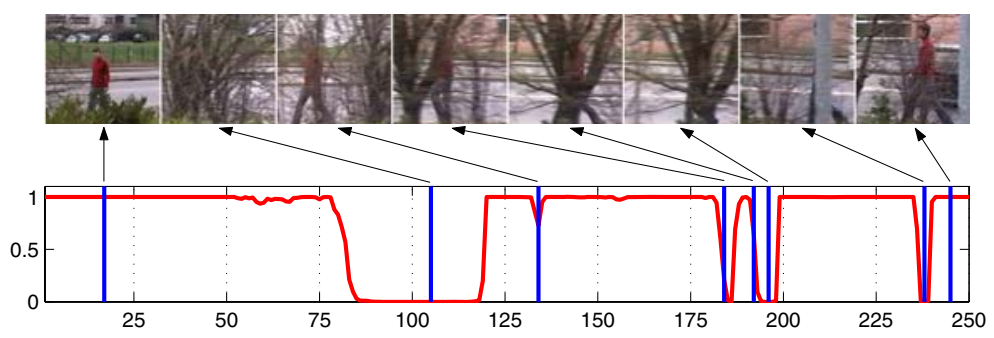

Fig. 3. Posterior visibility probability, $\xi_{1, t}=p\left(v_{t}=1 \mid \mathbf{y}^{t}\right)$, plotted against time for the example in Fig. 2. Selected key views show some occlusion and desocclusion events that respectively cause the visibility probability to drop, possibly to 0 , and to increase back to 1 .

Two data models,

$$
\begin{aligned}
& p\left(\mathbf{y}_{t} \mid \mathbf{x}_{t}, v_{t}=0\right)=p_{0}\left(\mathbf{y}_{t}\right) \\
& p\left(\mathbf{y}_{t} \mid \mathbf{x}_{t}, v_{t}=1\right)=p_{1}\left(\mathbf{y}_{t} \mid \mathbf{x}_{t}\right),
\end{aligned}
$$

will have to be specified, depending on whether the object of interest is visible in the image or not. In the former case, the likelihood is independent of the state value. Since our experiments are conducted in the context of color-based tracking we consider a simple observation model related to the more complex ones proposed in 94]. Pixel-wise location independent background and foreground models, $g_{0}$ and $g_{1}$, respectively, are specified over the selected color space. Assuming conditional independence of color measures over a sub-grid $S$ of pixels, we obtain

$$
\begin{aligned}
p_{0}\left(\mathbf{y}_{t}\right) & =\prod_{s \in S} g_{0}\left(\mathbf{y}_{s, t}\right) \\
p_{1}\left(\mathbf{y}_{t} \mid \mathbf{x}_{t}\right) & =\prod_{s \in R\left(\mathbf{x}_{t}\right)} g_{1}\left(\mathbf{y}_{s, t}\right) \prod_{s \in \bar{R}\left(\mathbf{x}_{t}\right)} g_{0}\left(\mathbf{y}_{s, t}\right),
\end{aligned}
$$

where $R\left(\mathbf{x}_{t}\right)$ is the image region associated with an object parameterized by the state $\mathbf{x}_{t}$, and $\mathbf{y}_{s, t}$ is the color at pixel $s$ in frame $t$.

For this dynamic model, the SMC architecture of Section 2.3 can be simplified. Indeed, the independence of the state evolution with respect to the auxiliary variables allows step $2 \mathrm{a}$ to be performed only $M$ times, and suggests the use of a unique proposal. A simple and classical choice is to take the state dynamics (22) as the proposal 22. We will adopt this approach here, while bearing in mind that any data-based proposal, including the optimal one in the rare cases that it is accessible, can be used in our generic framework. In case an object detector compliant with the tracking of interest is available, the proposal can also be chosen as a mixture combining the state dynamics and Gaussian distributions centered on detections (if any), as suggested in [7]. 
Fig. 2 shows results obtained on a sequence where a walking person is successfully tracked despite a succession of severe and total occlusions caused by trees in the foreground. The manual initialization ot the traker provides the reference foreground and background models defined as $5 \times 5 \times 5$ joint histograms in the RGB color space. The unknown state $\mathbf{x}_{t}$ comprises the position in the image plane $\left(n_{x}=2\right)$ and its dynamics (22) is taken to be a random walk with independent Gaussian noise with variance $10^{2}$ on each component. The parameters of the Markov chain on the visibility process are $\alpha_{01}=0.8$ and $\alpha_{10}=0.1$, and its initial distribution is given by $p\left(v_{0}=1\right)=0.8$. We use $N=200$ particles for the SMC implementation. The main quantities of interest are the marginal filtering distributions (5), which inform on the localization of the object of interest regardless of whether it is visible or not. The algorithm also recursively estimates the marginal visibility posterior $p\left(v_{t}=1 \mid \mathbf{y}^{t}\right)$. The time evolution of this quantity for the pedestrian sequence is plotted in Fig. 3. It correctly drops to zero for each complete occlusion of the tracked person. Note also that the ambiguity caused by less severe partial occlusions also results in small decreases in this quantity. Thus, besides its crucial role in the derivation of the recursive Bayesian filter for the augmented model, the estimation of the posterior visibility carries information that is interesting in its own right. Assessing the degree of occlusion of tracked objects is for instance a difficult and crucial problem when it comes to online updating of reference appearance models [6].

\subsection{Existence Process}

Using a Markovian binary variable to indicate presence in the scene is proposed in [9] to determine in a probabilistic fashion the beginning and end of the track for a single object. We adopt the same model here. However, sequential Monte Carlo is the only inference mechanism considered in [9], and it is conducted in the augmented state space. By comparison, our generic framework can be easily used with any Bayesian filtering technique and its SMC version implies sampling only in the object state space.

Following 9], we introduce a binary existence variable $e_{t}$ that indicates whether the object of interest is present $\left(e_{t}=1\right)$ or not $\left(e_{t}=0\right)$ in the scene at time $t$. The Markov chain prior on this binary variable is completely defined by the death and birth probabilities, $\alpha_{10}$ and $\alpha_{01}$. Conditional on the existence variables the state dynamics is specified by

$$
\begin{aligned}
& p_{00}\left(\mathbf{x}_{t} \mid \mathbf{x}_{t-1}\right)=p_{10}\left(\mathbf{x}_{t} \mid \mathbf{x}_{t-1}\right)=\delta_{\mathbf{u}}\left(\mathbf{x}_{t}\right) \\
& p_{01}\left(\mathbf{x}_{t} \mid \mathbf{x}_{t-1}\right)=p_{\text {init }}\left(\mathbf{x}_{t}\right) \\
& p_{11}\left(\mathbf{x}_{t} \mid \mathbf{x}_{t-1}\right)=p_{\text {dyn }}\left(\mathbf{x}_{t} \mid \mathbf{x}_{t-1}\right),
\end{aligned}
$$

where $\mathbf{u}$ is the consuming state that corresponds to the object not existing, $p_{\text {init }}$ is the initial state distribution, and $p_{\text {dyn }}$ is the object dynamic model. From the data model point of view, the existence process is similar to the visibility process. 

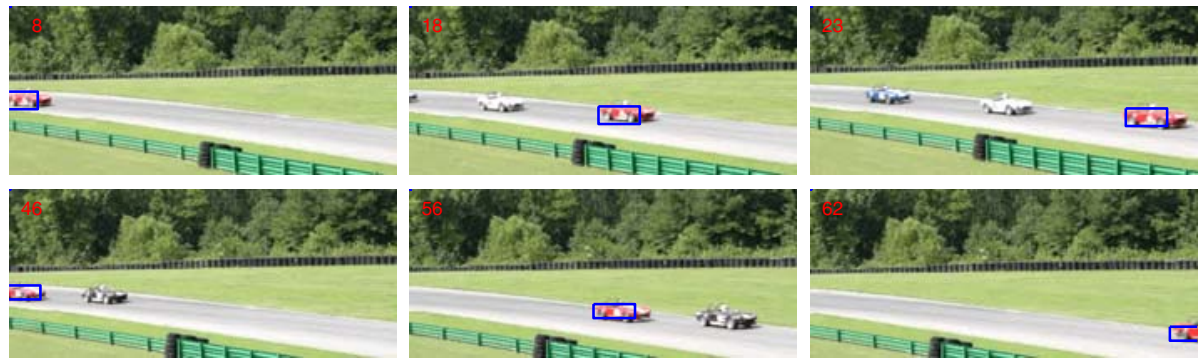

Fig. 4. Single object detection and tracking. The color model is initialized beforehand on one instance of a red car. The algorithm then successfully detects red cars that enter the scene, locks onto them and tracks them as long as they remain in view, and finally determines automatically when they disappear. In each of the displayed frames, the box amounts to the MC approximation of the posterior state expectation, conditional on existence $e_{t}=1$.

Due to the component (27) of the evolution model, non-existence $e_{t}=0$ deterministically forces $\mathbf{x}_{t}$ into fictitious state $\mathbf{u}$. This is carried over in the posterior model, yielding

$$
p_{0}\left(\mathbf{x}_{t} \mid \mathbf{y}^{t}\right)=\delta_{\mathbf{u}}\left(\mathbf{x}_{t}\right) .
$$

As a consequence, the algorithm only needs to recursively estimate the conditional filtering distribution for the case of the object existing, i.e., $p_{1}\left(\mathbf{x}_{t} \mid \mathbf{y}^{t}\right)$. Thus, within the SMC framework, only two proposal distributions, $q_{01}$ and $q_{11}$, are required, instead of four. As in the previous section, we only consider the simple case where these distributions coincide with their counterparts in the evolution model.

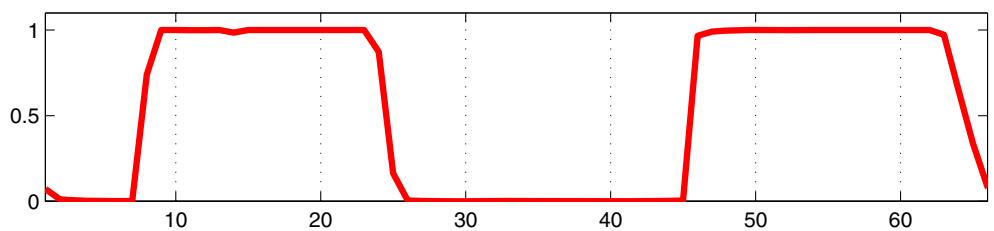

Fig. 5. Posterior existence probability, $\xi_{1, t}=p\left(e_{t}=1 \mid \mathbf{y}^{t}\right)$, plotted against time for the example in Fig. 4. When the object enters the scene, $\xi_{1, t}$ quickly ramps up to 1 , and falls back down to 0 when it exits.

In the following experiment, the observation model is defined as in the previous section. Yet again the state comprises the object location in the image plane, and in the state evolution model (28)-(29), $p_{\text {init }}$ and $p_{\text {dyn }}$ are respectively chosen as the uniform distribution over positions in the image plane and a random walk with independent Gaussian noise. The variance of the noise is $15^{2}$ for each 

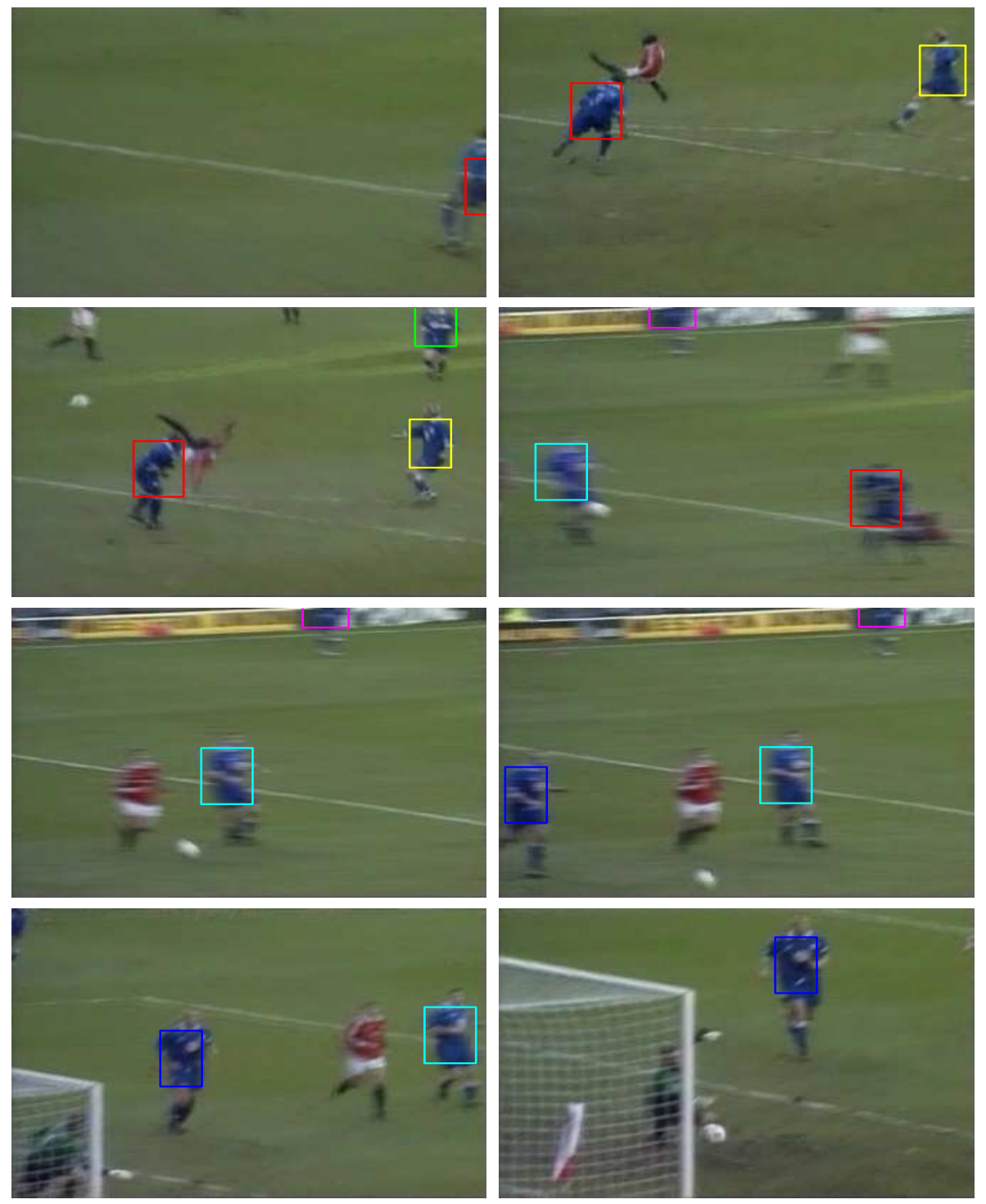

Fig. 6. Detection and tracking of multiple objects using multiple interacting trackers with existence process. Based on the same reference color model initialized beforehand on one blue player, six color-based trackers with existence process are run in parallel. They share at each instant the same proposal distribution $q_{01}$ which excludes regions already populated by "active" trackers (i.e, those with $\xi_{1, t}>0.2$ ). This simple system allows the proper detection and tracking of the blue players as long as they remain in view. The MC approximation of the posterior state expectation is displayed only for trackers that are "active" (i.e, those with $\xi_{1, t}>0.2$ ), using one color per tracker. 
component for the car race sequence in Fig. 4. Also, the state distribution at time $t=0$ coincides with $p_{\text {init }}$. Hence, contrary to the previous experiment, the tracker is not initialized manually at the beginning of the sequence (the reference foreground model is picked on an arbitrary red car in a different part of the video). For this experiment, the death and birth probabilities are respectively set to $\alpha_{01}=0.1$ and $\alpha_{10}=0.1$, and the initial existence distribution is given by $p\left(e_{0}=1\right)=0.1$. Finally, $N=50$ particles were sufficient to detect the entrance and exit of red cars in the field of view and to track them while present in the scene. Entrance and exit events are clearly identified by the variations in the posterior existence probability $\xi_{1, t}$, as shown in Fig. 5. In this example, a single tracker successively locks on to different cars, each one appearing in the image after the previous one has been successfully detected and tracked until disappearance. In practice, distinction between different tracked objects would be necessary, especially if they are likely to be present simultaneously in the image. In this context, the information carried by the existence probabilities facilitates the design of a mechanism that effectively initiates different trackers for each "detected" object and subsequently discards each tracker whose associated existence probability $\xi_{1, t}$ falls below a threshold.

An example of such a multiple object tracking is presented in Fig. 6, where football players from the same team are tracked in a video sequence. Due to player and camera movement the number of players in view varies continuously between 0 and 6 . Six trackers with existence process are run in parallel. Each of them locks on a different player as he enters the image and tracks him until he exits the field of view, at which point the tracker is disabled. In order to avoid that an "inactive" tracker becomes "active" by locking on an already tracked player, the proposal distribution $q_{01}$ is shared by all the trackers and reshaped at each instant. It is simply uniform over the part of the state space that is not yet occupied by any of the active trackers. Note, however, that we have not introduced any mechanism to handle mutual occlusions in this preliminary experiment.

\section{Conclusions and Perspectives}

In this paper we introduced a generic Bayesian filtering tool to perform tracking in the presence of a certain class of discrete auxiliary processes. The approach places no restriction on the ingredients of the evolution and observation models and on the selected type of filter (Kalman filter and its variants, particle filters). Hence the proposed framework allows re-use of existing architectures on a variety of tracking problems where the introduction of auxiliary discrete variables is useful. We demonstrated in particular how the technique can be applied in visual tracking to handle occlusions and object appearance/disappearance via visibility and existence binary processes. Experimental validation is presented in the particular case of color-based tracking with particle filters.

Our generic framework would now allow the combination of these two binary processes within a single tracking setup. This would amount to the manipulation 
of a joint auxiliary variable $\left(v_{t}, e_{t}\right)$ with three possible states, $(0,0),(0,1)$ and $(1,1)$. This would be especially useful to address the difficult problem of multiple object tracking where an unknown and varying number of objects of interest must be detected and tracked in presence of occlusions. In this case, however, mutual occlusions between the tracked objects might require a treatment different from the one of occlusions by other parts of the scene.

Other lines of future research concern the application of our generic framework to other types of tracking with auxiliary discrete processes, such as those with switches between different dynamics or different appearance models.

\section{References}

1. J. Giebel, D. Gavrila, and Schnörr. A Bayesian framework for multi-cue 3d object tracking. In Proc. Europ. Conf. Computer Vision, 2004.

2. M. Isard and A. Blake. Contour tracking by stochastic propagation of conditional density. In Proc. Europ. Conf. Computer Vision, 1996.

3. M. Isard and A. Blake. A mixed-state Condensation tracker with automatic modelswitching. In Proc. Int. Conf. Computer Vision, 1998.

4. M. Isard and J. MacCormick. BraMBLe: a Bayesian multiple-blob tracker. In Proc. Int. Conf. Computer Vision, 2001.

5. J. MacCormick and A. Blake. A probabilistic exclusion principle for tracking multiple objects. In Proc. Int. Conf. Computer Vision, 1999.

6. H. Nguyen, M. Worring, and R. van den Boomgaard. Occlusion robust adaptive template tracking. In Proc. Int. Conf. Computer Vision, 2001.

7. P. Perez, J. Vermaak, and A. Blake. Data fusion for visual tracking with particles. In Proc. IEEE 92(3):495-513.

8. K. Toyama and A. Blake. Probabilistic tracking in a metric space. In Proc. Int. Conf. Computer Vision, 2001.

9. J. Vermaak, P. Pérez, M. Gangnet, and A. Blake. Towards improved observation models for visual tracking: selective adaptation. In Proc. Europ. Conf. Computer Vision, 2002.

10. Y. Wu, T. Yu, and G. Hua. Tracking appearances with occlusions. In Proc. Conf. Comp. Vision Pattern Rec., 2003. 